\title{
Introduction to signal transduction ${ }^{*}$
}

\author{
Iva Greenwald ${ }^{\S}$, Howard Hughes Medical Institute and Department of \\ Biochemistry and Molecular Biophysics, Columbia University, College of \\ Physicians and Surgeons, New York, New York 10032 USA
}

Signal transduction is the means by which cells respond to extracellular information. The major signaling systems have been conserved to a remarkable extent in all animals. In this first edition of Wormbook, we present chapters describing most of the major pathways for which $C$. elegans has played critical roles in elucidating the components, function or regulation of signal transduction.

Much of the work described in these chapters illustrates the rubric, "the awesome power of C. elegans genetics". Since Sydney Brenner's initial description of C. elegans as a model organism, forward genetic approaches - screens for visible phenotypes and/or suppressors of mutant phenotypes - have identified many of the key components involved in signal transduction in all animals, and defined their diverse roles in development and cell physiology. More recently, the development of "reverse" genetic approaches that exploit the genomic sequence information, including RNA-mediated interference and screening methods to identify deletion alleles, has allowed additional signaling components to be identified and their roles to be determined. In sum, genetic analysis in $C$. elegans has illuminated the molecular mechanisms and biological roles of signaling systems, and has provided insights (or raised new questions) about their evolutionary origins.

We start with Gerard Manning's encyclopedia of the C. elegans "kinome" (see Genomic overview of protein kinases). Protein kinases direct many cellular processes and modify the activity and localization of many cellular proteins; their centrality has made them the subject of much work in C. elegans. There is tremendous diversity of protein kinases in the C. elegans genome: there are 439 protein kinase genes, and an additional 22 kinase fragments or pseudogenes. Manning also provides a comparative analysis featuring fungi, flies and humans, and reports, notably, that nematodes contain close homologs for $81 \%$ of all human kinases. Many of the key players described throughout the Signal transduction section are protein kinases.

Min Han and Paul Sternberg found in 1990 that Ras plays a pivotal role in vulval precursor cell specification in C. elegans, sparking a burgeoning field. Meera Sundaram's chapter on Receptor Tyrosine Kinase (RTK)-Ras-MAP kinase (MAPK) signaling (see RTKRas/MAP kinase signaling) features many different roles for RTK-Ras-MAPK signaling in C. elegans development. She has also described how genetic studies of Ras-mediated signaling in C. elegans have led to the first identification of many conserved core components and novel regulators of RTK-Ras signaling.

Cathy Savage-Dunn has reviewed roles of TGF- $\beta$; signaling in regulating dauer larva development, body size, and axon guidance (see TGF- $\beta$ signaling). She has also described the genetic identification of core components of this signaling pathway in $C$. elegans. The activated TGF- $\beta$ receptor is a heterodimer of two transmembrane serine/threonine kinase receptors. The activated receptor phosphorylates a Smad protein, which then forms a

\footnotetext{
*Edited by Lisa R. Girard. Last revised Feburary 29, 2005. Published September 9, 2005. This chapter should be cited as: Greenwald, I Introduction to signal transduction (September 9, 2005), WormBook, ed. The C. elegans Research Community, WormBook, doi/10.1895/wormbook.1.20.1, http://www.wormbook.org.

Copyright: () 2005 Iva Greenwald. This is an open-access article distributed under the terms of the Creative Commons Attribution License, which permits unrestricted use, distribution, and reproduction in any medium, provided the original author and source are credited

${ }^{\S}$ To whom correspondence should be addressed. E-mail: greenwald@ cancercenter.columbia.edu
} 
complex with another Smad protein to regulate transcription of target genes. Many Smad proteins were first identified through genetic analysis in $C$. elegans, and conventional and reverse genetic approaches in C. elegans continue to define new roles and to identify new components, regulators, and targets of TGF- $\beta$ signaling.

The Notch locus was defined genetically in Drosophila over half a century ago, and cell-cell interactions mediated by this receptor have been the subject of intense investigation in many systems. Genetic analysis in $C$. elegans has yielded several important "firsts" in understanding the mechanism and roles of LIN-12/Notch function as a receptor, including the role of LIN-12/Notch proteins in controlling binary cell fate decisions, cell autonomy and feedback modulation during cell fate decisions, conserved functional domains, and the mechanism of signal transduction. My chapter (see LIN-12/Notch signaling in C. elegans) is focused on how the "awesome power of $C$. elegans genetics" has identified many core components and modulators of LIN-12/Notch activity.

David Eisenmann has written a comprehensive overview of the many aspects of $C$. elegans development that are controlled by Wnt signaling (see Wnt signaling). Signal transduction by Wnt proteins has been widely viewed as involving a "canonical" pathway, in which signal transduction is mediated by the translocation of $\beta$-catenin from the cytosol into the nucleus, and a "non-canonical" pathway involving other signal transducers. However, in C. elegans, in some cases there also appear to be differences in the way components conventionally thought to act in one pathway operate, and a blurring of the distinction between the canonical and non-canonical pathways may prove to be more general.

G protein-coupled receptors (GPCRs) are the largest superfamily in animal genomes. A major class of GPCRs in mammals is olfactory receptors, as found in the seminal study by Linda Buck and Richard Axel ${ }^{\dagger}$. Hugh Robertson and James Thomas present an encyclopedia of putative chemoreceptors in C. elegans, which constitute an astonishing $7 \%$ of all predicted genes (see The putative chemoreceptor families of C. elegans). There are approximately 1200 potentially functional candidate chemoreceptor genes; there are also about 400 additional genes that, based on comparative genomics, appear to be pseudogenes in C. elegans but functional in other nematodes.

GPCRs transduce signals via heterotrimeric G proteins, composed of $\alpha, \beta$, and $\gamma$ subunits. The activated GPCR stimulates the exchange of GDP for GTP on the $\alpha$ subunit, causing the dissociation of G $\alpha$ from G $\beta \gamma$, either of which can interact with effectors. Hydrolysis of GTP restores the heterotrimer for another round of signaling. Carol Bastiani and Jane Mendel review the remarkably diverse roles of heterotrimeric G proteins in C. elegans neurobiology, behavior, and development, and the many powerful genetic approaches used to identify those roles as well as novel regulators (see Heterotrimeric G-proteins in C. elegans). They also describe roles for GPCR-independent pathways, in which other proteins facilitate the dissociation of G $\alpha$ and $G \beta \gamma$.

Erik Lundquist describes another large group of signal transducers that rely on GTP hydrolysis, small GTPases of the Ras superfamily (see Small GTPases). These signal transducers possess intrinsic GTPase activity that determines their GTP-bound versus GDP-bound state. They are key regulators of diverse cellular and developmental events. Many Ras-superfamily small GTPases are linked to transmembrane receptors; others regulate intracellular events. One of the principles that is emerging from genetic analysis of small GTPases in C. elegans is that many have overlapping, redundant roles. Lundquist also describes a striking example of how a study in $C$. elegans expedited the identification of a human disease gene.

Nuclear receptors (NRs) are transcription factors that, in the presence of their small lipophilic ligands, assemble coactivator complexes. Adam Antebi details how the NRs have undergone a dramatic expansion and divergence in the worm, which has six times the number of NR genes as in humans (see Nuclear hormone receptors in C. elegans). He has described how studies of NR function in C. elegans has illuminated their in vivo roles in development, physiology, and lifespan, as well as their place in global regulatory networks.

Thomas Bürglin and Patricia Kuwabara discuss the conservation and divergence of components of the Hedgehog signaling pathway in C. elegans (see Homologs of the Hh signalling network in C. elegans). Although $C$. elegans lacks a true Hedgehog ortholog, there are many Hedgehog-related genes, as well as other genes that encode proteins that participate in Hedgehog signaling. C. elegans also has multiple relatives of the Hedgehog receptor, Patched, as well as a single gene corresponding to the nuclear transcription factor Cubitus interruptus. However, the key signal transducer Smoothened and other components of the signal transduction cascade are "missing". Bürglin

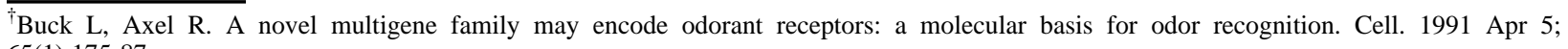
65(1):175-87. 
and Kuwabara discuss the evolutionary questions raised by this unusual complement of Hh pathway genes, and summarize what is known about the function of these genes in C. elegans.

Jonathan Ewbank describes the rapid progress that has been made identifying signal transduction pathways that mediate innate immunity and bacterial pathogenesis in C. elegans, a relatively new area of study (see Signaling in the immune response). These mediators including the Smads, MAP kinase, and PIP3, which are well-known for regulating other developmental or physiological processes as well. Ewbank discusses the involvement of these signaling pathways in diverse processes, and how the absence of some components or the divergence in function of others that have been implicated in innate immunity in vertebrates has mechanistic and evolutionary implications.

This collection of eleven chapters demonstrates that the study of signal transduction in C. elegans is a vibrant and diverse area. In addition to describing the mechanism and roles of signaling, the genetic analysis of signal transduction in $C$. elegans offers in vivo approaches to central problems in the signal transduction field: how the same signaling pathways elicit different responses in different contexts, and how different signaling inputs are integrated by a cell. Future updates will no doubt be concerned with these issues.

All WormBook content, except where otherwise noted, is licensed under a Creative Commons Attribution License. 